\title{
Perfil sociodemográfico e qualidade de vida de mulheres com doenças cardiovasculares e respiratórias: estudo de base populacional
}

\author{
Demographic profile and quality of life of women with cardiovascular and respiratory Diseases: \\ population-based study
}

Perfil sociodemográfico y calidad de vida de mujeres con enfermedades cardiovasculares $y$ respiratorias: estudio poblacional

Maria Cecília Moraes Frade', Camila Ferreira Leite ${ }^{2}$, Isabel Aparecida Porcatti de Walsh ${ }^{3}$, Gilberto Pereira Araújo ${ }^{4}$, Shamyr Sulyvan de Castro ${ }^{5}$

RESUMO | O objetivo do estudo é descrever o perfil de três grupos de mulheres: aparentemente saudáveis (não doentes-ND); com doenças cardiovasculares (DC); e com doenças respiratórias (DR) crônicas. A pesquisa comparou esses grupos conforme variáveis sociodemográficas (faixa etária, escolaridade, etnia e renda familiar) e dados sobre qualidade de vida (QV), segundo domínios: físico, psicológico, relações sociais e meio ambiente. Este estudo é caracterizado como quantitativo, analítico, observacional e transversal de base populacional, com informações extraídas do Inquérito de Saúde da Mulher, realizado na cidade de Uberaba-MG. Foram entrevistadas 1.387 mulheres, com o objetivo de coletar informações sobre DC, DR, idade, escolaridade, etnia e renda familiar. A QV foi estudada por meio do WHOQOL - Bref, segundo os quatro domínios. Foi realizado levantamento estatístico, descritivo e inferencial. Identificou-se que o grupo com DC é formado por mulheres com idade a partir de 50 anos e baixa escolaridade, em oposição ao grupo de mulheres com DR, que são mais jovens e possuem escolaridade superior. Quanto à QV, as mulheres com DC (31,65\%) apresentaram níveis inferiores em relação às mulheres com DR (19,10\%), nos domínios: físico (50,6;54,0;<0,0001), psicológico (55,1;58,7;<0,0001), relações sociais (75,8;77,2;0,0055) e meio ambiente (41,7;43,0;0,0173), valores em média. Portanto, o domínio de QV que obteve menor pontuação para todos os grupos foi o meio ambiente e as mulheres com DC apresentaram valores mais baixos, estatisticamente significativos, para todas as categorias.

Descritores | Doenças Crônicas Não Transmissíveis; Mulheres; Doenças Respiratórias; Doenças Cardiovasculares; Qualidade de Vida.

ABSTRACT | This study aims to describe the profile of three groups of women; apparently healthy (non-sick - NS); with cardiovascular diseases (CD); and with chronic respiratory diseases (RD). These groups were compared according to sociodemographic variables (age group, education, ethnicity, and family income) and quality of life (QoL) data, composing the domains: physical, psychological, social relations, and environment. This study is characterized as quantitative, analytical, observational, and cross-sectional populationbased, with information extracted from the Women's Health Survey, conducted in the city of Uberaba-MG. A total of 1,387 women were interviewed, and information on CD, RD, age, education, ethnicity and family income were collected. The QoL was studied using WHOQOL - BREF, according to its four domains. Descriptive and inferential statistics were performed. The CD group is composed of women aged 50 years and

'Universidade Federal de São Carlos (Ufscar) - São Carlos (SP), Brasil. E-mail: mariaceciliafrade@gmail.com. ORCID-0000-0002-0167-3114

${ }^{2}$ Universidade Federal do Ceará (UFC) - Fortaleza (CE), Brasil. E-mail: camilafl.fisio@gmail.com. ORCID-0000-0001-6375-8845

${ }^{3}$ Universidade Federal do Triângulo Mineiro (UFTM) - Uberaba (MG), Brasil. E-mail: isabelpwalsh@gmail.com. ORCID-0000-0002-2317-1326

${ }^{4}$ Universidade Federal do Triângulo Mineiro (UFTM) - Uberaba (MG), Brasil. E-mail: pereira.gilberto2012@gmail.com. ORCID-0000-0002-9149-6368

${ }^{5}$ Universidade Federal do Ceará (UFC) - Fortaleza (CE), Brasil. E-mail: castross@ufc.br. ORCID-0000-0002-2661-7899 
older and low education level, in contrast to the women of the RD group, who are younger and have higher education level. As for QoL, women with CD (31.65\%) had lower levels than women with RD (19.10\%), in the domains: physical (50.6;54.0;<0.0001), psychological (55.1;58.7;<0.0001), social relations (75.8;77.2;0.0055), and environment (41.7;43.0;0.0173), values on average and $p$, respectively. Therefore, the QoL domain that obtained the lowest score for all groups was the environment and women with CD presented lower values for all categories, which were statistically significant.

Keywords | Noncommunicable Diseases; Women; Respiratory Tract Diseases; Cardiovascular Diseases; Quality of Life.

RESUMEN I El objetivo del estudio es describir el perfil de tres grupos de mujeres: aparentemente sanas (no enfermas-NE); con enfermedades cardiovasculares (EC); y con enfermedades respiratorias crónicas (ER). La investigación comparó estos grupos según variables sociodemográficas (grupo de edad, escolaridad, etnia e ingresos familiares) y datos sobre calidad de vida (CV), según dominios: físico, psicológico, relaciones sociales y medio ambiente. Este estudio se caracteriza por ser cuantitativo, analítico, observacional y transversal de base poblacional, con información extraída de la Encuesta de Salud de la Mujer, realizada en la ciudad de Uberaba-MG. Se entrevistó a un total de 1.387 mujeres, con el objetivo de recopilar información sobre EC, ER, edad, escolaridad, etnia e ingresos familiares. La CV se estudió utilizando el WHOQOL - Bref, según los cuatro dominios. Se realizó encuesta estadística, descriptiva e inferencial. Se identificó que el grupo con EC está formado por mujeres con edad de 50 años o más y baja escolaridad, en contraposición al grupo de mujeres con EC, que son más jóvenes y tienen estudios superiores. En cuanto a la CV, las mujeres con EC (31,65\%) presentaron niveles más bajos en comparación con las mujeres con ER (19,10\%), en los siguientes dominios: físico (50,6; 54,0;<0,001), psicológico (55,1; 58,7;<0,0001), relaciones sociales $(75,8 ; 77,2 ; 0,0055)$ y medio ambiente $(41,7 ; 43,0$; 0,0173), valores medios. Por lo tanto, el el dominio de CV que obtuvo la puntuación más baja para todos los grupos fue el medio ambiente y las mujeres con EC presentaron valores más bajos, estadísticamente significativos, para todas las categorías.

Palabras clave | Enfermedades Crónicas No Transmisibles; Mujeres; Enfermedades Respiratorias; Enfermedades Cardiovasculares; Calidad de Vida.

\section{INTRODUÇÃO}

As doenças crônicas não transmissíveis (DCNT) caracterizam-se por serem de longa duração e por resultarem de uma combinação de fatores genéticos, fisiológicos, ambientais e comportamentais ${ }^{1}$. Entre as DCNT, as mais combatidas no panorama mundial são representadas pelas doenças cardiovasculares, diabetes, doenças respiratórias crônicas e cânceres ${ }^{2}$.

Mundialmente, com a evidente transição epidemiológica resultante do crescimento da expectativa de vida associada ao controle das doenças infecciosas e da mortalidade materno-infantil, as DCNT passaram a figurar como o problema dominante da saúde. Elas representam dois terços das causas totais de óbitos mundiais, metade das incapacidades e rápido crescimento de custos ${ }^{3}$, não só atribuíveis ao tratamento da doença em si, mas também aos gastos agregados a essas condições, como afastamentos do trabalho e perda de produtividade, com reflexos diretos na economia do país ${ }^{4}$. De acordo com o DATASUS 5 , os gastos hospitalares com doenças do aparelho circulatório foram cerca de $\mathrm{R} \$ 200$ milhões. Para as doenças do aparelho respiratório, foram gastos cerca de $\mathrm{R} \$ 91$ milhões por mês.
Acompanhando os esforços globais para controle das DCNT, o Ministério da Saúde lançou, em 2011, o "Plano de Ações Estratégicas para o Enfrentamento das DCNT" para 2011-2022, assumindo metas e compromissos fundamentados em ações populacionais para controlar os índices elevados dessas doenças ${ }^{6}$. No ano de 2013, a Pesquisa Nacional de Saúde, conduzida no Brasil para monitorar as DCNT, investigando tanto fatores de risco, quanto morbidades associadas, revelou que $45,1 \% \mathrm{da}$ população brasileira tem, ao menos, uma DCNT e esse percentual eleva-se a 50,4\% entre as mulheres ${ }^{5}$.

Diante das dificuldades econômicas frente ao cenário brasileiro e da importância do papel da mulher na sociedade, destaca-se a relevância em conhecer algumas características sociodemográficas e indicadores de qualidade de vida (QV) de mulheres que hoje apresentam DCNT. A pesquisa contempla, em particular, as DCNT de causas cardiovasculares e respiratórias, devido à sua alta incidência. Sabendo que as políticas públicas para o enfrentamento dessas doenças agem em caráter preventivo, entende-se que elas precisam atingir uma parcela da população não doente, mas já exposta aos fatores de risco ou com hábitos de vida similares. Dessa forma, o objetivo deste estudo é descrever e comparar o perfil de mulheres aparentemente saudáveis com mulheres portadoras de doenças cardiovasculares ou doenças 
respiratórias crônicas, segundo variáveis sociodemográficas e de QV.

\section{METODOLOGIA}

Este é um estudo quantitativo, analítico, observacional e transversal de base populacional, com informações extraídas do Inquérito de Saúde da Mulher (ISA-Mulher), de 2015, realizado na cidade de Uberaba-MG.

As voluntárias incluídas no estudo foram mulheres a partir de 18 anos de idade, que concordaram e assinaram o termo de consentimento livre e esclarecido (TCLE). O processo de seleção foi realizado por amostragem probabilística em múltiplos estágios. O cálculo amostral foi feito e considerado o mínimo necessário com 1.530 mulheres, de acordo com estudo prévio ${ }^{7}$. Esse estudo utilizou um intervalo de confiança de $95 \%$ e uma margem de erro de $2,5 \%$, de acordo com a fórmula $\left(n=\frac{\left(Z_{a}\right)^{2} \pi(1-\pi)}{(m e)^{2}}\right)$, sendo que $Z_{a}$ : pontuação da distribuição normal com um nível de significância $(=0,05) ;$ : margem de erro para estimativas de intervalo. Para o atual estudo, a amostra foi de 1.387 mulheres, 170 foram excluídas por apresentarem dados faltantes.

As coletas foram realizadas por entrevistadoras treinadas, em visitas domiciliares, no período de março a outubro de 2015 , com checagem aleatória de $10 \%$ via telefone. As informações sobre doenças cardiovasculares e respiratórias foram coletadas de forma autorreferida. Dessa forma, a informação foi aceita pelo entrevistador diante do relato da respondente de que um médico havia feito o diagnóstico nosológico da doença indicada.

As condições de saúde estudadas foram agrupadas, compondo uma variável dicotômica relativa à presença de pelo menos uma doença. Elas foram divididas em DC hipertensão arterial, doença coronariana/angina pectoris, infarto, insuficiência cardíaca, cardiomegalia ou outras - e $\mathrm{DR}$ - infecções repetidas do trato respiratório, bronquites e sinusites crônicas, asma, enfisema, tuberculose pulmonar e outras. As participantes foram agrupadas de acordo com a faixa etária (<30 anos; 30-39; 40-49; 50-69; e $\geq 70$ ); a escolaridade foi categorizada em analfabeto; até 5 anos de estudo; $>5-<9 ; 9-<12 ; \geq 12$; a etnia foi autorreportada e classificada como branca; preta/parda; e outra; e a renda familiar per capita foi categorizada em até 0,5 salários mínimos (SM); >0,5-1 SM; >1-2,5 SM; e >2,5 SM.

A QV foi estudada por meio do WHOQOL Bref, instrumento da Organização Mundial da Saúde
(OMS), validado para uso no Brasil ${ }^{8}$. Essa ferramenta foi desenvolvida especificamente para avaliação da QV, sendo composta por 26 questões com respostas em escala de Likert de 1 a 5. O WHOQOL - Bref gera escores que variam de 0 a 100, apresentados por domínios (físico, meio ambiente, relações sociais e psicológico) ou como escore geral, sendo que quanto maior a pontuação, melhor a $\mathrm{QV}$.

Inicialmente, foi criada uma máscara no programa EpiData ${ }^{\circledR}$ para a entrada dos dados, previamente à tabulação, com dupla digitação. A análise deste estudo consistiu em estatística descritiva, por meio de frequências simples e relativas, médias e desvios-padrão. Respeitando a normalidade e homogeneidade dos dados, a estatística inferencial usou Anova one-way, com pós-teste de Tukey para a comparar os domínios de QV e os grupos. Também foi utilizado um teste de comparação de proporções (teste qui-quadrado com análise de resíduo) para comparar os dados faixa etária, escolaridade, etnia e renda familiar per capita, segundo os grupos, considerando-se o nível de significância de 5\%.

\section{RESULTADOS}

Nesta pesquisa, foram entrevistadas 1.557 mulheres e encontradas prevalências de $28,19 \%$ (439 mulheres) com DC, $17,02 \%$ (265 mulheres) com DR, 10,92\% (170 mulheres) com doenças cardiovasculares e respiratórias e 43,87\% (683 mulheres) sem doenças de causa respiratória ou cardiovascular (ND - não doentes; dados não apresentados nas tabelas). Mulheres com duplo acometimento (cardíaco e respiratório) não entraram nas análises. Dessa forma, a amostra final deste estudo é de 1.387 mulheres.

A Tabela 1 mostra a distribuição geral das variáveis de faixa etária, escolaridade, etnia e renda em cada grupo de mulheres estudadas. No que diz respeito à faixa etária, percebe-se um comportamento significativamente diferente entre grupos, sendo que nas mulheres com DC e ND tem-se maior proporção nas faixas etárias mais elevadas (acima de 50 anos) em relação ao geral. Nas mulheres com DR, identifica-se uma maior proporção de mulheres mais jovens (abaixo de 30 anos).

Quanto à escolaridade, no grupo de mulheres com $\mathrm{DC}$, houve uma maior prevalência de mulheres com menor escolaridade (até 5 anos). Nos grupos DR e ND, há maior escolaridade (12 ou mais). A etnia prevalente foi branca para todos os grupos. Com relação à renda per capita predominante das mulheres estudadas, foi de 0,5 a um salário mínimo, não sendo um fator determinante de diferenças estatísticas (Tabela 1). 
Tabela 1. Distribuição de frequência das variáveis explicativas faixa etária, escolaridade, etnia e renda familiar per capita, segundo os grupos de mulheres com doenças cardiovasculares, respiratórias e de não doentes, Uberaba, 2015

\begin{tabular}{|c|c|c|c|c|c|c|c|c|c|}
\hline \multirow{3}{*}{ Faixa etária (anos) } & \multicolumn{6}{|c|}{ GRUPOS } & & & \multirow[b]{3}{*}{ p-valor } \\
\hline & \multicolumn{2}{|c|}{ DC (439 - 31,65\%) } & \multicolumn{2}{|c|}{ DR (265 - 19,10\%) } & \multicolumn{2}{|c|}{ ND $(683-49,24 \%)$} & \multicolumn{2}{|c|}{ GERAL (1387 - 100,0\%) } & \\
\hline & $\mathrm{n}$ & $\%$ & $\mathbf{n}$ & $\%$ & $\mathrm{n}$ & $\%$ & $\mathbf{n}$ & $\%$ & \\
\hline Menor que 30 & 15 & $3,4^{+}$ & 79 & $29,8^{+}$ & 155 & $22,7^{+}$ & 249 & 18,0 & $<0,0001^{*}$ \\
\hline 30 a 39 & 14 & $3,2^{+}$ & 61 & $23,0^{+}$ & 145 & $21,2^{+}$ & 220 & 15,9 & \\
\hline 40 a 49 & 38 & $8,7^{+}$ & 54 & $20,4^{+}$ & 128 & $18,7^{+}$ & 220 & 15,9 & \\
\hline 50 a 69 & 247 & $56,3^{+}$ & 67 & $25,3^{+}$ & 214 & $31,3^{+}$ & 528 & 38,1 & \\
\hline 70 ou mais & 125 & $28,5^{+}$ & 4 & $1,5^{+}$ & 41 & $6,0^{+}$ & 170 & 12,3 & \\
\hline Escolaridade (anos) & $\mathrm{n}$ & $\%$ & $n$ & $\%$ & $\mathrm{n}$ & $\%$ & $\mathbf{n}$ & $\%$ & p-valor \\
\hline Analfabeto & 27 & $6,2^{+}$ & 2 & $0,8^{+}$ & 17 & $2,5^{+}$ & 46 & 3,3 & $<0,0001^{*}$ \\
\hline Até 5 & 152 & $34,6^{+}$ & 22 & $8,3^{+}$ & 97 & $14,2^{+}$ & 271 & 19,5 & \\
\hline$>5 a<9$ & 106 & 24,1 & 52 & 19,6 & 165 & 24,2 & 323 & 23,3 & \\
\hline $9 a<12$ & 53 & $12,1^{+}$ & 74 & $27,9^{+}$ & 155 & $22,7^{+}$ & 282 & 20,3 & \\
\hline 12 ou mais & 101 & $23,0^{+}$ & 115 & $43,4^{+}$ & 249 & $36,5^{+}$ & 465 & 33,5 & \\
\hline Etnia & $\mathrm{n}$ & $\%$ & $\mathrm{n}$ & $\%$ & $\mathrm{n}$ & $\%$ & $\mathrm{n}$ & $\%$ & p-valor \\
\hline Branca & 267 & $61,2^{+}$ & 149 & 56,7 & 350 & $51,5^{+}$ & 766 & 55,5 & $0,008^{*}$ \\
\hline Preta/Parda & 158 & $36,2^{+}$ & 103 & 39,2 & 314 & $46,2^{+}$ & 575 & 41,7 & \\
\hline Outra & 11 & 2,5 & 11 & 4,2 & 16 & 2,4 & 38 & 2,8 & \\
\hline Renda familiar per capita & n. & $\%$ & $\mathbf{n}$ & $\%$ & $\mathrm{n}$ & $\%$ & $\mathbf{n}$ & $\%$ & p-valor \\
\hline Até 0,5 salário mínimo & 96 & 21,9 & 59 & 22,3 & 187 & 27,4 & 342 & 24,7 & 0,09 \\
\hline >0,5 a 1 salário mínimo & 167 & 38,0 & 87 & 32,8 & 224 & 32,8 & 478 & 34,5 & \\
\hline >1 a 2,5 salários mínimos & 114 & 26,0 & 85 & 32,1 & 198 & 29,0 & 397 & 28,6 & \\
\hline >2,5 salários mínimos & 62 & 14,1 & 34 & 12,8 & 74 & 10,8 & 170 & 12,3 & \\
\hline
\end{tabular}

Fonte: Inquérito de Saúde da Mulher, realizado na cidade de Uberaba-MG

n: número de mulheres; *: p<0,05 a variável explicativa apresenta distribuição significativa segundo os grupos de mulheres; ': análise de resíduo - diferença significativa em relação à proporção geral.

As médias e desvios-padrão dos domínios de QV são apresentados na Tabela 2.

Tabela 2. Distribuição das médias e desvios-padrão dos domínios de qualidade de vida, segundo ocorrência de doenças respiratórias, cardiovasculares e em mulheres sem essas doenças, Uberaba, 2015

$\begin{array}{ccccc}\begin{array}{c}\text { Domínios de } \\ \text { Qualidade Vida }\end{array} & \begin{array}{c}\text { DC } \\ (n=439)\end{array} & \text { DR }(n=265) & \text { ND }(n=683) & p \\ \text { Físico } & 50,56 \pm 11,2 & 53,98 \pm 10,4^{+} & 55,99 \pm 10,1^{* \S} & <0,0001 \\ \text { Psicológico } & 55,07 \pm 8,68 & 58,69 \pm 9,16^{+} & 57,73 \pm 8,56^{*} & <0,0001 \\ \text { Relações sociais } & 75,8 \pm 14,4 & 77,2 \pm 14,9 & 78,6 \pm 14,4^{*} & 0,0055 \\ \text { Meio ambiente } & 41,73 \pm 6,63 & 43 \pm 6,63^{+} & 42,72 \pm 6,63^{*} & 0,0173\end{array}$

Fonte: Inquérito de Saúde da Mulher, realizado na cidade de Uberaba-MG

Os dados foram apresentados em média e desvio-padrão. DC: doenças cardiovasculares; DR: doenças respiratórias; ND: não doentes; " : diferença estatística entre DCXND; ; : diferença estatística entre DCXDR; s: diferença estatística entre DRXND.

\section{DISCUSSÃO}

Em síntese, entre as mulheres entrevistadas, $31,65 \%$ possuíam DC; $19,10 \%$ DR; e 49,24\% sem doenças respiratórias ou cardiovasculares (ND). A faixa etária predominante das mulheres com DC e ND foi a partir de 50 anos, já as mulheres com DR eram mais jovens. As mulheres com DC apresentaram baixa escolaridade em relação às mulheres com $\mathrm{DR}$ ou ND. A respeito da QV, o domínio que obteve menor pontuação para todos os grupos foi o meio ambiente, ao passo que, ao comparar os grupos, as mulheres com DC apresentaram valores mais baixos para todas as categorias, com diferença estatística para todos os domínios. $\mathrm{Na}$ amostra de mulheres deste estudo, identificou-se que doenças crônicas cardiovasculares foram mais prevalentes que doenças crônicas respiratórias. Silva et al. ${ }^{9}$ objetivou caracterizar o perfil de morbidade referida por usuários das equipes de saúde da família na macrorregião nordeste de Minas Gerais. Eles encontraram porcentagens de morbidade referida no aparelho circulatório e respiratório de 37,1 e 25,4 para as mulheres, corroborando os dados encontrados neste estudo. Esse predomínio de DCNT de causa cardiovascular n o estado mineiro é reflexo do cenário nacional, visto que a Pesquisa Nacional de Saúde de 2013 revelou que cerca de um quinto da população nacional tem diagnóstico médico de hipertensão arterial ${ }^{4}$.

Com relação à faixa etária das mulheres que reportaram DCNT, houve prevalência das DC e ND em mulheres com idades entre 50 e 69 , destacando o predomínio dessa condição de saúde em faixas etárias mais elevadas. A hipertensão arterial, por exemplo, frequentemente, surge nas mulheres com o avançar da idade, sendo que a perda da proteção estrogênica pós-menopausa pode ter um 
papel fundamental nessa patologia ${ }^{10}$. Além disso, na pósmenopausa existe também um aumento da formação de placa de ateroma e de inflamação, fator que contribui com o desenvolvimento da aterosclerose ${ }^{11}$.

Em conjunto, essas condições têm reflexos diretos em desfechos bastante desfavoráveis (doenças cerebrovasculares e cardíacas), que associam-se a elevadas taxas de mortalidade, endossando que a alta prevalência dessas doenças precisa ser combatida com políticas públicas efetivas.

A respeito da escolaridade, as mulheres com DC apresentaram menores níveis de escolaridade no presente estudo. Já em pesquisa realizada com população israelita, identificou-se que o fator escolaridade influencia o risco de mortalidade por doenças cardiovasculares ${ }^{12}$. No estudo, observaram que há um risco de mortalidade duas vezes maior entre as mulheres de 45 a 69 anos com menos de oito anos de escolaridade ao comparar com mulheres que possuíam 13 anos de escolaridade. Além disso, os autores encontraram interações significativas em relação à idade e ao sexo, indicando que o efeito da educação sobre a mortalidade foi mais forte entre as mulheres em comparação com os homens e entre os indivíduos mais jovens em comparação com os mais velhos. Reforça-se o impacto de se trabalhar com estratégias de educação em saúde com amostras de sujeitos de baixa renda e escolaridade, numa tentativa de oportunizar o acesso a informações e influenciar sobremaneira as taxas de DCNT de origem cardiovascular.

O ISA encontrou maior porcentagem de mulheres brancas, seguidas por negras/pardas e outras, em todos os grupos (ND, DC e DR), com diferença estatística entre os grupos DC e ND. O estudo da Vigilância de Fatores de Risco e Proteção para Doenças Crônicas por Inquérito Telefônico (Vigitel), em todos os estados do Brasil,realizado em 2012, entrevistou 45.448 pessoas. Desse total, 40,9\% eram brancos, $38,0 \%$ pardos e $8,5 \%$ pretos, resultado semelhante ao encontrado nesta pesquisa ${ }^{13}$. Porém, ao analisar as diferenças segundo raça/cor entre os fatores de risco, de proteção de DCNT e de doenças relatadas como hipertensão arterial, há maior prevalência nos negros e pardos, tanto em mulheres, quanto nos homens, mesmo quando ajustados os fatores socioeconômicos e demográficos, como escolaridade e idade ${ }^{13}$.

Com relação aos escores de QV obtidos pelo instrumento WHOQoL Bref, foi observado que o domínio meio ambiente apresentou menor pontuação em todos os grupos. Isso demonstra menor QV nesse aspecto, semelhante ao encontrado para grupo de voluntários sem doenças crônicas ${ }^{14}$. O perfil socioeconômico da amostra deste estudo pode justificar esse achado, tendo em vista que o domínio meio ambiente do instrumento WHOQoL aborda questões relacionadas a recursos financeiros, oportunidades de lazer, segurança, e demais situações favorecidas em cenários econômicos mais vantajosos, que provavelmente não são oportunizadas às mulheres entrevistadas, visto que elas apresentaram renda média de 0,5 a um salário mínimo.

Ao considerarmos que as mulheres com DR obtiveram maior pontuação nos domínios psicológico e meio ambiente, ao serem comparadas com o grupo $\mathrm{ND}$, duas situações precisam ser analisadas. A primeira, já mencionada, acerca da correspondência da situação socioeconômica à $\mathrm{QV}$ relativa ao domínio meio ambiente (a amostra com DR foi a que apresentou maiores níveis de escolaridade e renda). A segunda situação especulativa considera que as mulheres agrupadas no grupo ND têm sabidamente descartadas as doenças crônicas de causas respiratórias e cardiovasculares, mas outras doenças, que poderiam ter impacto negativo na QV relacionada a esse domínio específico, podem estar presentes.

O domínio físico do WHOQoL teve, significativamente, pior escore no grupo de mulheres com DC, quando comparado ao grupo com DR. Um estudo conduzido no Nordeste brasileiro analisou a relação das limitações funcionais com as condições sociodemográficas e de saúde de mulheres idosas de baixa condição econômica. A pesquisa concluiu que o aumento da idade, a condição de viuvez, a presença de hipertensão arterial, insatisfação com a saúde, a ausência de práticas de atividades físicas de lazer são fatores determinantes para as limitações funcionais das mulheres idosas de baixa renda ${ }^{15}$. Considerando o perfil da população estudada, as políticas públicas com medidas preventivas para DCNT que objetivarem controlar os fatores de risco dessas morbidades precisam ser bastante direcionadas e efetivas ao perfil da população. As estratégias adotadas precisam atingir a população de baixa renda e escolaridade, apresentando melhorias no serviço de saúde, particularmente com qualificação da atenção básica. $\mathrm{O}$ controle dessas doenças terá reflexo não só na obtenção de uma população mais saudável, mas também terá impacto sobre o desenvolvimento macroeconômico do país, dado o expressivo impacto financeiro dessas morbidades ${ }^{16}$.

Para a amostra do estudo já portadora de DCNT de causas cardiovasculares e pulmonares, são indicados programas de reabilitação cardíaca ${ }^{17}$ e pulmonar ${ }^{18}$, objetivando ganhos funcionais e de QV para esses 
indivíduos. Dada a indisponibilidade de número suficiente de serviços públicos ambulatoriais, estratégias de reabilitação domiciliar de baixo custo têm demonstrado resultados favoráveis, no sentido de alcançarem ganhos funcionais comparáveis aos obtidos nas estratégias clássica de reabilitação supervisionada, além de diminuírem as barreiras relacionadas à adesão aos programas ${ }^{19}$.

Por fim, para completa interpretação dos resultados deste estudo, é preciso considerar que a falta de um diagnóstico médico; a ausência de quantificação da gravidade da doença, seu tempo de duração e a ausência de informações acerca das condições de tratamento aplicadas a cada situação específica representam limitações para a prevenção e o tratamento.

\section{CONCLUSÃO}

Dessa forma, conclui-se que a prevalência de doenças cardiovasculares e respiratórias em mulheres do município de Uberaba é de $31,65 \%$ e $19,10 \%$, respectivamente. As mulheres com DC, predominantemente, têm idade a partir de 50 anos e baixa escolaridade, todavia, há um perfil oposto entre as com DR. Além disso, as mulheres com DC possuem pior QV em relação ao grupo de DR e ND e o domínio que obteve menor pontuação para todos os grupos foi o meio ambiente.

\section{REFERÊNCIAS}

1. World Health Organization. Noncommunicable diseases [Internet]. Geneva: WHO; 2018 [cited 2018 Jun 1]. Available from: http://www.who.int/en/news-room/fact-sheets/detail/ noncommunicable-diseases

2. World Health Organization. Global status report on noncommunicable diseases 2010. Geneva: WHO; 2011.

3. Ali MK, Jaacks LM, Kowalski AJ, Siegel KR, Ezzati M. Noncommunicable diseases: three decades of global data show a mixture of increases and decreases in mortality rates. Health aff (Millwood). 2015;34(9):1444-55. doi: 10.1377/ hlthaff.2015.0570.

4. Malta DC, Stopa SR, Szwarcwald CL, Gomes NL, Silva JB Jr, Reis AAC. Surveillance and monitoring of major chronic diseases in Brazil-National Health Survey. Rev Bras Epidemiol. 2015;18(2):316. doi: 10.1590/1980-5497201500060002.

5. Datasus. Morbidade hospitalar do SUS [Internet]. Rio de Janeiro: Coordenação Geral de Disseminação de Informações em Saúde; 2018 [cited 2021 Jul 30]. Available from: http://tabnet.datasus. gov.br/cgi/tabcgi.exe?sih/cnv/niuf.def
6. Duncan BB, Chor D, Aquino EML, Bensenor IM, Mill JG, Schmidt MI, et al. Chronic non-communicable diseases in Brazil: priorities for disease management and research. Rev Saude Publica. 2012;46(1):126-34. doi: 10.1590/S0034-89102012000700017.

7. Henriques IF, Walsh IAP, Meirelles MCCC, Pereira GA, Castro SS. Relation of common mental disorder, physical activity and body mass index in women, population-based study. J Phys Educ. 2017;28(1):e2819. doi: 10.4025/jphyseduc.v28i1.2819.

8. Fleck MP, Louzada S, Xavier M, Chachamovich E, Vieira G, Santos $L$, et al. Application of the Portuguese version of the abbreviated instrument of quality life WHOQOL-bref. Rev Saude Publica. 2000;34(2):178-83. doi: 10.1590/s0034-89102000000200012.

9. Silva VM, Pereira IVS, Rocha MJL, Caldeira AP. Morbidity in users of family health teams in the northeast of Minas Gerais based on the International Classification of Primary Care. Rev Bras Epidemiol. 2014;17(4):954-67. doi: 10.1590/1809-4503201400040013.

10. Di Giosia P, Giorgini P, Stamerra CA, Petrarca M, Ferri C, Sahebkar A. Gender differences in epidemiology, pathophysiology, and treatment of hypertension. Curr Atheroscler Rep. 2018;20(3):13. doi: 10.1007/s11883-018-0716-z.

11. Meyer MR, Fredette NC, Howard TA, Hu CJ, Chinnasamy R, Amann K, et al. G Protein-coupled estrogen receptor protects from atherosclerosis. Sci Rep. 2014;4(1):7564. doi: 10.1038/ srep07564.

12. Brannon L, Updegraff JA, Feist J. Health psychology : an introduction to behavior and health. Wadsworth: Cengage Learning; 2010.

13. Malta DC, Moura L, Bernal RTI. Differentials in risk factors for chronic non-communicable diseases from the race/ color standpoint. Cien Saude Colet. 2015;20(3):713-25. doi: 10.1590/1413-81232015203.16182014.

14. Tüzün H, Aycan S, IIIhan MN.Impact of Comorbidity and Socioeconomic Status on Quality of Life in Patients with Chronic Diseases who Attend Primary Health Care Centres. Cent Eur J Public Health. 2015;23(3):188-94. doi: 10.21101/cejph.a3990.

15. Virtuoso JS Jr, Guerra RO. Fatores associados às limitações funcionais em idosas de baixa renda. Rev Assoc Med Bras. 2008;54(5):430-5. doi: 10.1590/S0104-42302008000500017.

16. Abegunde DO, Mathers CD, Adam T, Ortegon M, Strong K. The burden and costs of chronic diseases in low-income and middle-income countries. Lancet. 2007;370(9603):1929-38. doi: 10.1016/S0140-6736(07)61696-1.

17. Hamm LF, Wenger NK, Arena R, Forman DE, Lavie CJ, Miller TD, et al. Cardiac rehabilitation and cardiovascular disability. J Cardiopulm Rehabil Prev. 2013;33(1):1-11. doi: 10.1097/ HCR.0b013e31827aad9e.

18. Silva CCBM, Xavier RF, Carvalho CRF. Reabilitação pulmonar no Brasil. Fisioter Pesqui. 2017;24(4):347-8. doi: 10.1590/1809-2950/00000024042017.

19. Neves LF, Reis MH, Gonçalves TR. Home or communitybased pulmonary rehabilitation for individuals with chronic obstructive pulmonary disease: a systematic review and meta-analysis. Cad Saude Publica. 2016;32(6):e00085915. doi: 10.1590/0102-311X00085915. 